\title{
Ação Multifatorial do Quimioterápico Antineoplásico Capecitabina
}

\author{
Multifactor Action of Antineoplastic Chemotherapy Capecitabine
}

\author{
Vitor Alexandre Pezolato ${ }^{1}$ \\ Bruno Ferreira Gonçalves e Silva² \\ Carlos Alberto da Silva ${ }^{3}$
}

\section{RESUMO}

Objetivo: Analisar o glicogênio muscular, o perfil hematológico e as alterações eletrocardiográficas de ratos tratados com o medicamento a base de capecitabina. Metodologia: Foram utilizados ratos da linhagem Wistar, com 3 a 4 meses de idade e peso de 180 a 200 gramas da $A N I L A B \cap$, sem restrição alimentar, com temperatura constante de $23 \pm 2^{\circ} \mathrm{C}$ e ciclo normal de dia e noite, com 12 horas. O medicamento foi administrado por gavagem em uma dose de $359 \mathrm{mg} / \mathrm{kg}$, dissolvida em tampão de citrato $40 \mathrm{mM}$, contendo $5 \%$ de goma arábica como transporte. Resultados: Foi obsrvado que ocorreu diferença entre os grupos experimentais, sendo que os animais tratados com capecitabina tiverem concentrações menores de glicogênio hepático e muscular, além do medicamento diminuir os parâmetros hematológicos, resultando em alterações eletrocardiográficas importantes. Conclusão: O medicamento modifica a homeostasia metabólica tecidual e altera a reatividade cardiovascular tanto no padrão adrenérgico quanto colinérgico.

\section{DESCRITORES}

Capecitabina. Glicogênio. Eletrocardiografia. Glicogênio Hepático.

\begin{abstract}
Objective: Analyze muscle glycogen, hematological profile and electrocardiographic changes in rats treated with a capecitabine drug. Methodology: Wistar rats aged of 3 to 4 months and weighing 180 to 200 grams of $A N I L A B^{\circledR}$, without food restriction, with a constant temperature of $23 \pm 2^{\circ} \mathrm{C}$ and normal cycle of 12 hours during the day and night. The medication was administered by gavage in a dose of $359 \mathrm{mg} / \mathrm{kg}$, dissolved in $40 \mathrm{mM}$ citrate buffer, containing $5 \%$ arabic gum as transport. Results: It was seen that there was a difference between the experimental groups, with the animals treated with capecitabine having lower concentrations of hepatic and muscular glycogen, in addition to the medication reducing the hematological parameters, resulting in important electrocardiographic changes. Conclusion: Thus, this drug modifies metabolic tissue homeostasis and alters cardiovascular reactivity in both the adrenergic and cholinergic pattern.
\end{abstract}

\section{DESCRIPTORS}

Capecitabine. Glycogen. Electrocardiography. Hepatic glycogen.

${ }^{1}$ Docente das Faculdades Integradas Einstein de Limeira (FIEL) e do Programa de Graduação em Fisioterapia, Limeira, São Paulo, Brasil.

${ }^{2}$ Docente da Universidade Paulista (UNIP) do Programa de Graduação em Fisioterapia, Campinas, São Paulo, Brasil.

${ }^{3}$ Graduado em Medicina Veterinária pela Universidade Paulista (UNIP), Campinas, São Paulo, Brasil. 
câncer é uma doença que teve uma incidência de 582.590 mil pessoas no ano de 2018 e resultou na morte de 197.698 no mesmo ano. Essa doença pode ser caracterizada pelo rápido e incontrolável crescimento celular, com a possibilidade de se espalhar pelos tecidos do corpo' ${ }^{1}$.

As células possuem capacidades de se replicarem formando novos tecidos ou reparando lesões estruturais, sendo que ao término de sua funcionalidade metabólica a mesma irá iniciar o processo de apoptose ${ }^{2}$.

Uma célula se torna cancerígena quando seu sinal de apoptose não é respondido, assim sua capacidade proliferativa pode se tornar agressiva ao ponto de acontecer alterações genéticas, suficientes, ao ponto desta célula ter a capacidade de invasão tecidual, também conhecido como metástase. As células neoplásicas, por se replicarem muito mais rápido que células saudáveis, ainda, sendo inespecíficas, o seu metabolismo é alto e para sustentar essa necessidade metabólica ocorre a neoangiogenese ao seu redor ${ }^{3}$.

A capecitabina é um medicamento composto, cujo precursor de 5-floruracil (5FU), que continua a ser essencial no tratamento de tumores, realizando sua atividade, principalmente, em neoplasias malignas do trato gastrointestinal, colorretais, de cabeça e pescoço, mama, ovário, próstata, fígado e do trato geniturinário 4 .

Uma vez o medicamento sendo administrado, a capecitabina é, inicialmente, convertida em 5-desoxi-5-fluorouridina (5-DFUR) pela carbozilesterase hepática, a qual é convertida em 5'-desoxi-5-fluorouridina (5'DFUR) pela citidina desaminase, ambos ocorrendo no fígado ${ }^{5,6}$. Finalmente, a 5'DFUR é convertida em 5-FU pela timidina-fosforilase, cuja atividade é aumentada em células cancerígenas ${ }^{5}$.

A atividade biológica do 5-FU está associada ao seu metabolismo, devido à formação de monofosfato de fluorodeoxiuridina (FdUMP) que é um metabólico que age impedindo a enzima timidilato sintetase (TS) e, consequentemente, a síntese de DNA, levando a célula à apoptose ${ }^{6}$.

O 5-FU também é convertido em monofosfato de fluorouridina (FUMP), o qual pode ser submetido a duas fosforilações, passando a trifosfato de fluorouridina (FUTP), que tem a capacidade de ser incorporado no RNA no lugar da uridina-5-trifosfato (UTP), interferindo no processamento do RNA, sendo que a citoxicidade do 5-FU está relacionada com a sua função de afetar a replicação do RNA e da inibição da enzima TS ${ }^{4}$.

Desta maneira, as vias metabólicas do 5-FU, as quais o seu mecanismo de ação não interfere somente nas células cancerígenas, mas no tecido periférico também, o objetivo do presente trabalho visou observar as respostas elétricas cardíacas, metabólicas e hematológicas de ratos submetidos ao tratamento medicamentoso com o quimioterápico a base de capecitabina ${ }^{7}$.

\section{METODOLOGIA}

Foram utilizados ratos da linhagem Wistar (Rathus novergicus var, albinus, Rodentia, Mamalia), com 3 a 4 meses de idade com peso de 180 a $200 \mathrm{~g}$ da ANILABß localizada na Cidade de Paulínia - SP, os quais receberam água e alimentação a vontade ( $a d$ libitum), sendo mantidos em ambiente com temperatura constante de $23 \pm 2{ }^{\circ} \mathrm{C}$ e ciclo 
claro/escuro de 12 horas, com luz acesa a partir das 6 horas, sendo mantidos em gaiolas coletivas contendo no máximo 5 animais e distribuídos em dois grupos $(n=10)$, sendo um controle $(C)$ e outro tratado com capecitabina $(\mathrm{X})$.

O projeto foi aprovado pela CEUA UNIMEP, por meio do protocolo $\mathrm{n}^{\circ}$. 09/2017.

Tratamento com capacetabina

A capecitabina (Xeloda ${ }^{\circledR}$, F. Hoffman La - Roche, Basle Suíça) foi administrada por meio de gavagem em dose terapêutica $359 \mathrm{mg} / \mathrm{kg}$ (2/3 da dose considerada tóxica), dissolvida em tampão de citrato $40 \mathrm{mM}(\mathrm{pH}$ $6,0)$ contendo $5 \%$ de goma arábica como veículo durante 7 dias.

Análise eletrocardiográfica

Para a avaliação das ondas e intervalos eletrocardiográficos, os ratos foram anestesiados com pentobarbital sódico (40 $\mathrm{mg} / \mathrm{kg} / \mathrm{ip}$ ), mantidos em posição supina, com respiração espontânea para registro do ECG. Os eletrodos foram conectados aos canais do computador (Heart Ware System) e registradas três derivações bipolares (DI, DII e DIII) e nas três derivações amplificadas ( $a V R$, aVL e aVF) com sensibilidade $2 \mathrm{~N}$ e velocidade de $50 \mathrm{~mm} /$ segundo. O intervalo QT foi medido em dez batimentos consecutivos, do início do complexo QRS ao ponto de retorno da onda $\mathrm{T}$ isoelétrica definido como segmento TP. O intervalo QT foi corrigido pela frequência cardíaca usando a fórmula de Bazett (QTc = $Q T / \sqrt{ } R R$ ) e posteriormente foi calculada a dispersão do intervalo QTc, subtraindo o intervalo
QTc mais curto do QTc mais longo (QTcd = QTc Max - QTc min). As análises foram realizadas por um único avaliador para minimizar as divergências na medida da dispersão.

Glicogênio hepático e muscular

Após a anestesia e a eutanásia realizada a partir do decapitamento por guilhotina, amostras do fígado e dos músculos sóleo (S), gastrocnêmio branco (GB) e vermelho (GV), ventrículo esquerdo $(V)$ e diafragma $(D)$ foram retiradas, digeridas em $\mathrm{KOH} 30 \%$ a quente por 60 minutos, em seguida foi adicionado $\mathrm{Na}_{2} \mathrm{SO}_{4}$ e etanol absoluto para permitir a precipitação do polímero. As amostras foram centrifugadas a 300 rpm por 15 minutos, sendo que o sobrenadante foi descartado e o precipitado foi ressuspendido em água destilada. Assim, foi adicionado etanol absoluto para realização de uma nova centrifugação. Para a hidrólise ácida do glicogênio, foi adicionado fenol e realizada a leitura em espectrofotômetro em $505 \mathrm{~nm}$. Os valores foram expressos em $\mathrm{mg} / 100 \mathrm{mg}$ de peso úmido.

Dosagem de ácidos graxos livres (AGL)

O método consistiu na extração do AGL por meio de um solvente seletivo, onde o extrato foi tratado com uma mistura reagente que contendo nitrato de cobre, ocorrendo, em consequência disto, a formação de um sabão de cobre. O metal assim ligado reagiu com o dietilditilcarbamato, produzindo uma coloração cuja intensidade é diretamente proporcional ao teor de AGL que é medida espectrofotometricamente em $435 \mathrm{~nm}$.

Análise de hematócrito, hemoglobina, 
eritrometria e leucometriaForam realizadas as contagens de hematócritos, hemoglobina, eritrometria e leucometrtia por meio de técnicas rotineiras de laboratório hematológico.

Foi realizada a contagem de hemácias pela câmara de Neubauer. O método para contagem global das células sanguíneas consistiu geralmente em diluir o sangue, em proporção conhecida, com o líquido diluidor Hayem, permitindo a conservação das células em estudo. Foi realizado de acordo com 7 etapas: (1) Pipetou-se $4 \mathrm{ml}$ do líquido diluidor em um tubo de ensaio; (2) Transferiu-se os $0,02 \mathrm{ml}$ de sangue para o tubo com o líquido diluidor, lavando com ele o interior da ponteira por aspiração e expulsão do líquido. A diluição foi de 1:200; (3) Agitou-se suavemente por inversão para uma correta homogeneização; (4) Com uma pipeta preencheu-se os retículos da câmara de contagem, evitando excesso de líquido e bolhas de ar sob a lamínula aderida firmemente à câmara; (5) Deixou-se repousar por dois minutos para sedimentação dos glóbulos; (6) Focalizou-se a preparação com pequeno aumento no microscópio para localizar o retículo e observar a distribuição uniforme das hemácias. Posteriormente, observou-se com aumento de 100X ou 400X, conforme necessidade; (7) Foi feita a contagem de todas as hemácias encontradas nos quadros marcados em "H".

Para a contagem de leucócitos: (1) Pipetou-se 0,4 ml do líquido de Turk no tubo; (2) Com pipeta automática, pipetou-se 0.02 $\mathrm{ml}$ de sangue; (3) Foi transferido os $0,02 \mathrm{ml}$ de sangue para o tubo com o líquido diluidor, lavando com ele o interior da pipeta por aspiração e expulsão do líquido. A diluição foi de 1:20; (4) Agitou-se suavemente por dois minutos; (5) Encheu-se os dois retículos da câmara de contagem, evitando excesso de líquido e bolhas de ar sob a lamínula, aderida firmemente à câmara por compressão daquela sobre esta, cobrindo ambos os retículos; (6) Deixou-se repousar de um a dois minutos para sedimentação dos glóbulos; (7) Foi focalizado a preparação com pequeno aumento no microscópio para localizar.

Análise estatística

Os dados coletados foram tabulados e posteriormente analisados pelo software estatístico "GraphPad Prism 8". A análise estatística foi procedida pela aplicação do teste Shapiro-Wilk para verificação da normalidade. Os dados que se demonstraram paramétricos passaram pela análise de variância de uma via (one-way ANOVA), quando foi o caso, de duas vias (two-way ANOVA), ambas seguido do post-hoc teste de Tukey. Em todos os cálculos foi fixado um nível crítico de $p<0,05$ para significância estatística.

\section{RESULTADOS}

As reservas glicogênicas são importantes por representarem o status de resistência metabólica dos organismos, desta forma, pequenas reservas indicam redução na eficiência funcional e maior propensão à fadiga. Por sua vez, grandes reservas indicam maior eficiência metabólica. A análise das reservas hepáticas de glicogênio indicou que o grupo tratado com capecitabina apresentou reservas $68,3 \%$ menores se comparado ao grupo controle (não tratado). A concentração hepática de glicogênio $(\mathrm{mg} / 100 \mathrm{mg})$ de ratos 
controle (C) e tratados com capecitabina (X) (dose $359 \mathrm{mg} / \mathrm{kg}$ ). Os valores correspondem a média \pm desvio padrão (dp), $\mathrm{n}=10$. (Figura 1).

Dentro do perfil metabólico também foram avaliadas as concentrações glicogênicas na porção branca e vermelha do músculo gastrocnêmico e no músculo sóleo, verificando-se que o grupo tratado com capecitabina (X) apresentou menores reservas, sendo observados valores menores $67 \%$, 56\% e $45 \%$, respectivamente, se comparado ao controle. A concentração muscular de glicogênio (mg/100mg) de ratos controle (C) e tratados com capecitabina (X; dose $359 \mathrm{mg} / \mathrm{kg}$ ) dos músculos gastrocnêmio porção branca (GB), gastrocnêmio porção vermelha (GV), sóleo (S), no ventrículo (V) e diafragma (D). Os valores correspondem a média $\pm d p, n=10$ (Figura 2).

A seguir, o estudo foi direcionado à avaliação dos parâmetros hematológicos, visto sua importância para manutenção do equilíbrio homeostático.

Dentro do perfil hematológico foi observado que muitos parâmetros foram alterados devido ao tratamento, iniciando pela série vermelha. Neste contexto, o hematócrito do grupo tratado apresentou-se reduzido em $19 \%$, condição também observada na hemoglobina, cuja concentração foi $17,8 \%$ menor no grupo tratado e, por fim, na eritrometria em que também foi observado comprometimento, alcançando valores $63,5 \%$ menores em decorrência do tratamento (Tabela 1).

No que se refere à série branca, a determinação da leucometria também indicou ser menor no grupo tratado, verificando-se valores $35 \%$ menores (Tabela 1 ).

Sabendo-se que os organismos apresentam ajustes quimiometabólicos e estes

Figura 1. Concentração hepática de glicogênio

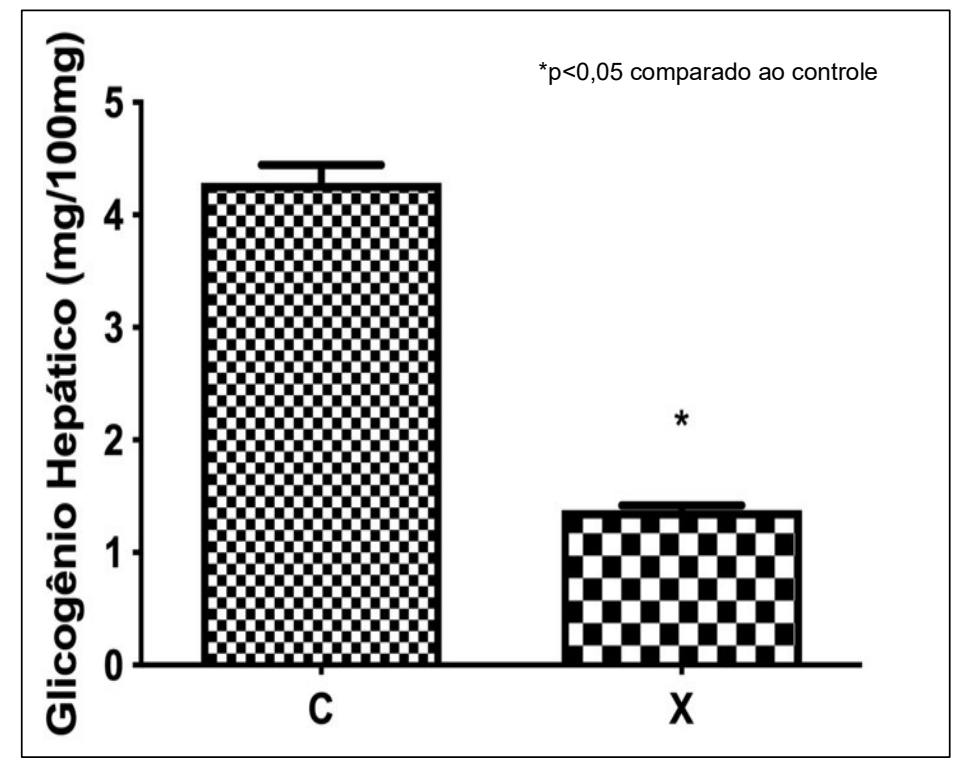


Figura 2. Concentração muscular de glicogênio

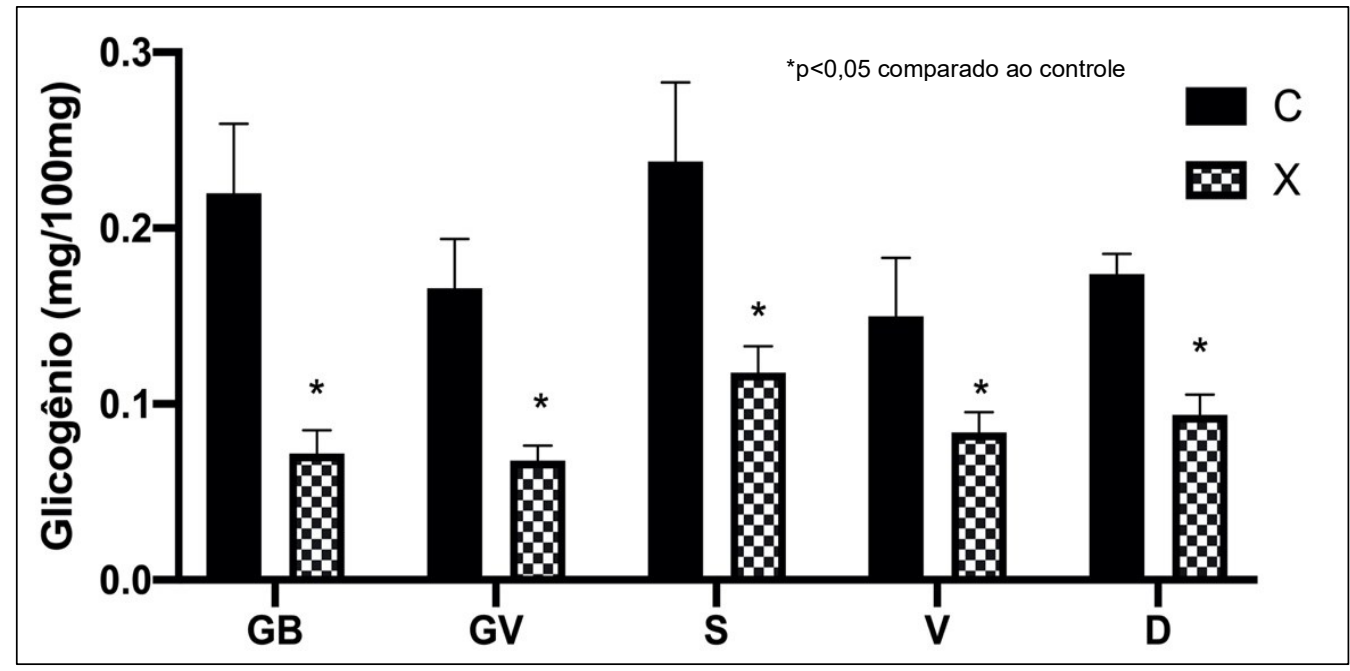

Tabela 1. Parâmetros hematológicos de ratos controle $(C)$ e tratados com capecitabina

\begin{tabular}{ll|l|c|c|c}
\hline Hematócrito (\%) & $\begin{array}{c}\text { Hemoglobina } \\
(\mathrm{g} / \mathrm{dL})\end{array}$ & $\begin{array}{c}\text { Eritrometria } \\
(\text { milhões })\end{array}$ & $\begin{array}{c}\text { Leucometria } \\
(\mathrm{mil})\end{array}$ & $\begin{array}{c}\text { AGL } \\
(\mathrm{mmol} / \mathrm{L})\end{array}$ \\
\hline \multicolumn{5}{c}{$\begin{array}{c}\text { Média } \pm \mathrm{DP} \\
(\mathrm{n}=10)\end{array}$} \\
\hline $\mathrm{C}$ & $41,3 \pm 0,2$ & $14 \pm 0,5$ & $7,4 \pm 0,8$ & $4,48 \pm 0,4$ & $203 \pm 8$ \\
\hline $\mathrm{X}$ & $33,5 \pm 2,5^{*}$ & $11,5 \pm 0,4^{*}$ & $2,7 \pm 0,2^{*}$ & $2,9 \pm 0,2^{*}$ & $297 \pm 10$ \\
\hline
\end{tabular}

Tabela 2. Frequência cardíaca e parâmetros dos intervalos e segmentos das ondas eletrocardiográficas $(\mathrm{ms})$ de ratos

\begin{tabular}{|c|c|c|c|c|c|c|}
\hline & $\begin{array}{c}\mathrm{FC} \\
\text { (bat/min.) }\end{array}$ & $\begin{array}{l}\text { QRS } \\
\text { (ms) }\end{array}$ & $\begin{array}{l}\mathrm{QT} \\
(\mathrm{ms})\end{array}$ & $\begin{array}{l}\text { QTc } \\
\text { (ms) }\end{array}$ & $\begin{array}{l}\text { Inter. PR } \\
\text { (ms) }\end{array}$ & $\begin{array}{l}\text { Segm. PR } \\
\text { (ms) }\end{array}$ \\
\hline \multicolumn{7}{|c|}{$\begin{array}{l}\text { Média } \pm \text { DP } \\
(n=6)\end{array}$} \\
\hline $\mathrm{C}$ & $240,9 \pm 11$ & $51 \pm 3,1$ & $110,9 \pm 5,8$ & $233,40 \pm 16$ & $33 \pm 4,3$ & $15 \pm 2,2$ \\
\hline$X$ & $288,2 \pm 11^{*}$ & $39,4 \pm 4,3^{*}$ & $99 \pm 9,5$ & $247,8 \pm 26^{*}$ & $57 \pm 2,4^{*}$ & $26 \pm 4,0$ \\
\hline
\end{tabular}

${ }^{*} p<0,05$ comparado ao controle. 
têm relação direta com o status nutricional ou ação de fármacos, foi avaliada a concentração plasmática de ácidos graxos livres que representa o status gerado pela condição sine qua non. Nesta análise foi avaliada a concentração plasmática de ácido graxo livre sendo verificado que o grupo tratado com capecitabina apresentou concentrações $46 \%$ maiores representado por $203,21 \pm 8 \mathrm{mmol} / \mathrm{L}$ na condição controle e $297,13 \pm 10 \mathrm{mmol} / \mathrm{L}$ no grupo tratado. Os parâmetros hematológicos de ratos controle $(\mathrm{C})$ e tratados com capecitabina (X; dose 359mg/kg). Tabela 1.

A análise eletrocardiográfica iniciou-se avaliando a frequência cardíaca, seguindo a regra da diferença entre o pico de duas ondas $\mathrm{R}$ consecutivas. Foram observados valores médios $20 \%$ maiores no grupo tratado $(X)$, de acordo com a Tabela 2.

A seguir foi avaliado o intervalo QRS, que corresponde à passagem do sinal elétrico pelo feixe de His e pelas fibras de Purkinge promovendo a despolarização ventricular, sendo verificado que o intervalo foi $41,1 \%$ maior no grupo $(\mathrm{X})$ e ainda no traçado eletrocardiográfico foi verificado supra desnivelamento. Ainda, foi observado diferença estatisticamente significativa nos valores do Intervalo PR, que corresponde ao tempo entre a onda $\mathrm{P}$ e o início do complexo QRS, sendo indicativo da velocidade de condução do impulso elétrico desde o nodo atrioventricular até aos ventrículos. Neste caso, o grupo (X) apresentou valores $70,5 \%$ maior em relação ao grupo controle. Os demais dados analisados pelo eletrocardiograma não demonstraram diferenças estatísticas. A frequência cardíaca (FC, bat/min) e parâmetros dos intervalos e segmentos das ondas eletrocardiográficas (ms) de ratos controle (C) e medicados com capecitabina (X; dose 359mg/kg). (Tabela 2 ).

No aspecto metabólico cardíaco foi avaliada a reserva de glicogênio do ventrículo esquerdo e do diafragma, sendo verificado que no ventrículo essas reservas apresentaram valores de concentração 46,6\% menores no grupo tratado $(X)$ enquanto no diafragma as concentrações foram de $47,05 \%$, também menores. (Figura 2).

\section{DISCUSSÃO}

Muitas substâncias químicas (fármacos) utilizadas no tratamento de diversas patologias, em especial o câncer, são indutoras de expressivas modificações nas funções orgânicas predispondo a múltiplas sintomatologias e desajustes ${ }^{8}$. Tais modificações envolvem ações tanto do sistema endócrino quanto do sistema nervoso autônomo. Assim, a literatura mostra que as modificações decorrem de ações associadas entre diferentes hormônios, a saber: hormônio adrenocorticotrófico (ACTH), hormônio somatotrófico (GH - hormônio de crescimento), hormônio tireotrófico (TSH), glicocorticoides das suprarrenais e adrenalina (catecolamina). Uma vez que estes hormônios apresentam intensa ação lipolítica, considera-se que o aumento na concentração plasmática de ácido graxo livre (AGL) indica a ação destes, mobilizando as reservas de triglicerídeos dos adipócitos e com isso oferecendo maiores concentrações de AGL aos tecidos ${ }^{9}$.

Esses desajustes indicam a implantação de uma condição estressora desencadeada pela circulação do fármaco, acompanhando uma condição clínica denominada fadiga 
oncológica ${ }^{10,11}$. Os dados são notórios em demonstrar que o tratamento com capecitabina gera alterações no metabolismo hepático, uma vez que, por ser citotóxico pode atuar também na homeostasia dos hepatócitos, lócus de primeira passagem do fármaco ${ }^{12}$. A análise das reservas glicogênicas hepáticas mostra menores reservas no grupo tratado, indicando ação direta da capecitabina nas vias sinalizadoras que formam tais reservas, considerando ainda que o epitélio intestinal possa ser sensibilizado pela mesma e apresentar redução na dinâmica de captação de glicose, indicando um efeito indireto do quimioterápico.

No mesmo aspecto de análise foi verificado que as reservas glicogênicas musculares também foram reduzidas, com maior expressão no músculo gastrocnêmio (fibra branca, glicolítica) que também apresenta as maiores reservas de glicogênio dentre os demais tipos de fibras, reiterando o comprometimento na formação de tais reservas. Cabe ressaltar que a literatura mostra que a resistência à insulina ocorre no tratamento com quimioterápicos ${ }^{13}$. Este dado pode refletir o sintoma de cansaço manifestado por pacientes sob tratamento.

Certo é que os músculos também podem utilizar ácido graxo livre como substrato metabólico, o qual, uma vez disponível no plasma e sendo lipossolúvel, é captado pelos músculos como fonte substrato energético. Neste contexto, a análise das reservas musculares glicogênicas reflete uma das vias metabólicas que predominam e podem ser comprometidas pela possível resistência insulínica gerada pela capecitabina. Ainda no aspecto estrutural, pode-se considerar que tem sido descrito sarcopenia nos pacientes em tratamento com capecitabina, sendo um fator adicional de comprometimento e reflexo das menores reservas energéticas ${ }^{14-16}$.

Do mesmo modo que ocorre a fraqueza muscular, devem ser consideradas as alterações nos parâmetros hematológicos ${ }^{17}$. Os dados mostraram que ocorreu redução no número de células sanguíneas, condição que acompanha a sugestão inserida na bula do fabricante, porém não discrimina os tipos de células afetadas ${ }^{18}$.

A Agência Nacional de Vigilância Sanitária (ANVISA) também indica que o tratamento com capecitabina gera depressão da atividade da medula óssea alterando a homeostasia e comprometendo a gênese das células sanguíneas ${ }^{19}$.

A literatura já caracterizou o status de anemia como número baixo dos glóbulos vermelhos (hemácias), pequena concentração de hemoglobina e menor percentual de eritrócitos $^{20}$. A maioria das pessoas com anemia sentem-se cansadas ou fracas, mesmo sintoma comum de pacientes em tratamento com o quimioterápico capecitabina, indicando comprometimento na formação das células sanguíneas ${ }^{21,22}$.

Os dados mostraram que todos os parâmetros hematológicos foram expressivamente comprometidos pelo tratamento com capecitabina, representado pela redução na eritrometria, no hematócrito bem como na concentração de hemoglobina e, desta forma, o presente estudo corrobora que a capecitabina reduz a eficiência na geração das células sanguíneas pela medula óssea ${ }^{23}$.

Estudos ligados a fisiologia/farmacologia cardiovascular têm sugerido expressivas alterações induzidas por quimioterápicos cardiotóxicos antimetabólitos, os quais com- 
prometem a atividade cardíaca ${ }^{24}$. Na década de 2000 , era consenso que a incidência e os fatores de risco de cardiotoxicidade associada à capecitabina ainda não estavam definidos ${ }^{25}$. Em 2009, os mesmos autores descreveram que o quimioterápico capecitabina promoveu isquemia miocárdica, condição que desencadeia um reflexo na via autonômica simpática moduladora da atividade do nódulo sino atrial promovendo aumento na frequência cardíaca, condição que referenda a presente pesquisa em um expressivo aumento na frequência cardíaca. Cabe ressaltar que, frente à intensa modificação na frequência cardíaca, ocorrem expressivas modificações compensatórias na condutância das vias intracardíacas enquanto fator de proteção para o músculo cardíaco e ainda promovem desajustes metabólicos visto as menores reservas glicogênicas ventriculares e diafragmáticas ${ }^{26}$.

As maiores evidências científicas resultam de relatos de caso sugerindo e reiterando as associações entre o tratamento com capecitabina e disfunções cardíacas ${ }^{27}$. Este estudo mostra que ocorreram alterações nos segmentos QRS, QTc e IPR, indicando redução compensatória na distribuição do sinal elétrico no músculo cardíaco buscando minimizar modificações impostas pelo tratamento com capecitabina. Ainda, pode-se observar nos dados aqui apresentados que o tratamento além de ser indutor de isquemia também está associado aos efeitos significativos sobre a reatividade cardiovascular adrenérgica e a inervação parassimpática ${ }^{28}$.

Um ponto merecedor de destaque é que foi observado tanto supra-desnivelamento quanto infra-desnivelamento do segmento ST acompanhando de modificações nos parâmetros das ondas eletrocardiográficas indicando cardiotoxicidade no tratamento com capecitabina.

\section{CONCLUSÃO}

O tratamento com capecitabina além promover comprometimento nos parâmetros hematológicos, modifica a homeostasia metabólica tecidual e altera a reatividade cardiovascular tanto no padrão adrenérgico quanto colinérgico. Estes dados visam socializar informações que possam servir de orientação a profissionais da saúde no intuito de buscar uma monitorização mais efetiva da anamnese ao longo do curso de tratamento.

\section{REFERÊNCIAS}

1. INCA, Instituto Nacional de Câncer. O que é Câncer [acesso em 12 abr 2019].

2. INCA, Instituto Nacional de Câncer. Números de Câncer [acesso em 12 abr 2019].

3. Oliveira PA, Colaço A, Chaves R, Guedes-Pinto H, De-La-Cruz PLF, Lopes C. Chemical carcinogenesis. An Acad Bras Ciênc. 2007; 79:593-616.

4. Breda M, Barattè S. A review of analytical methods for the determination of 5-fluorouracil in biological matrices. Anal Bioanal Chem. 2010; 397:1191-201.

5. Ishikawa T, Sekiguchi F, Fukase Y, Sawada N, Ishitsuka H. Positive correlation between the efficacy of capecitabine and doxifluridine and the ratio of thymidine phosphorylase to dihydropyrimidine dehydrogenase activities in tumors in human cancer xenografts. Cancer Res. 1998; 58:685-690. 
6. Malet-Martino M, Jolimaitre P, Martino R. The prodrugs of 5-fluorouracil. Curr Med Chem Anticancer Agents. 2002; 2:267-310.

7. Shamim HM, Minami Y, Tanaka D, Ukimori S, Murray JM, Ueno M. Fission yeast strains with circular chromosomes require the 9-1-1 checkpoint complex for the viability in response to the anti-cancer drug 5-fluorodeoxyuridine. PLoS ONE. 2017; 12:e0187775.

8. Martins I, Rosa HVD. Considerações Toxicológicas da Exposição Ocupacional aos Fármacos Antineoplásicos. Rev Bras Med Trab. 2004; 2:118-125.

9. Picon RMP, Cosner AF, Silveira RO. Relação entre estressores, estresse e ansiedade. Rev Psiquiatr. 2003; 25:65-74.

10. Eicher MV. A sinalização celular: um enfoque no papel do AMPc no mecanismo de ação do glucagon e epinefrina no fígado. Revista UNINGÁ. 2006; 7:187-194.

11. Borges JA, Quintão MMP, Chermont SSMC, Mendonça FiIho HTF, Mesquita ET. Fadiga: Um Sintoma Complexo e seu Impacto no Câncer e na Insuficiência Cardíaca. Int J Cardiovasc Sci. 2018; 31:433-442.

12. Lunardi D, Zart D, Fasolin T, Gonçalves CBT. Atenção Farmacêutica para pacientes em uso de Capecitabina. Rev Bras Farm. 2009; 90:250-257.

13. Nicolini A, Giardino R, Carpi A, Ferrari P, Anselmi L, Colosimo S, Conte M, Fini M, Giavaresi G, Berti P, Miccoli P. Metastatic breast cancer: an updating. Biomed Pharmacother. 2006; 60:548-556.

14. Prado CM, Baracos VE, McCargar LJ, Reiman T, Mourtzakis M, Tonkin K, Mackey JR, Koski S, Pituskin E, Sawyer MB. Sarcopenia as a determinant of chemotherapy toxicity and time to tumor progression in metastatic breast cancer patients receiving capecitabine treatment. Clin Cancer Res. 2009; 15:2920-2926.

15. Walko CM, Lindley C. Capecitabine: a review. Clin Ther. 2005; 27:23-44.

16. Ebner N, D. Anker S, Von Haehling S. Recent developments in the field of cachexia, sarcopenia, and muscle wasting: highlights from the 11th Cachexia Conference. J cachexia sarcopenia muscle. 2019; 10:218-225.

17. Martins TL, Pinto AR, Pires JMD, Silva MJS, Couto DHN, Calil-Elias S. Reação adversa induzida por capecitabina: a importância da farmacovigilância. Rev Bras Farm. 2013; 4:24-26.
18. Xeloda [Bula]. Rio de Janeiro: Roche Produtos Químicos e Farmacêuticos; 2016 [acesso em 02 dez 2020].

19. ANVISA. Agência Nacional de Vigilância Sanitária. Cobertura: medicamento capecitabina (xeloda); 2016 [acesso em 02 dez 2020].

20. Azevedo MRA. Hematologia Básica: Fisiopatologia e Diagnóstico Laboratorial. 5. Ed. São Paulo: Revinter, 2012.

21. Hamilton A, Hortobagyi G. Chemotherapy: What Progress in the Last 5 Years? J Clin Oncol, 2005; 23.

22. Newman NB, Sidhu MSNK, Baby R, Moss RA, Nissenblatt MJ, Chen T, Shou-EnLu Jabbour SK. Long-Term Bone Marrow Suppression During Postoperative Chemotherapy in Rectal Cancer Patients After Preoperative Chemoradiation Therapy. Int J Radiat Oncol Biol Phys. 2016; 94.

23. Siddiqui NS, Godara A, Byrne MM, Saif MW. Capecitabine for the treatment of pancreatic cancer. Expert Opin Pharmacother. 2019; 20:112-119.

24. Kalil Filho R, Haijar LA, Bacal F, Hoff PM, Diz M del P, Galas FRBG, et al. I Diretriz Brasileira de Cardio-Oncologia da Sociedade Brasileira de Cardiologia. Arq Bras Cardiol. 2011; 96:1-52.

25. Yeh ET, Tong AT, Lenihan DJ, Yusuf SW, Swafford J, Champion $\mathrm{C}$, et al. Cardiovascular complications of cancer therapy: diagnosis, pathogenesis, and management. Circulation. 2004; 109:3122-3131.

26. Yeh ET, Bickford CL. Cardiovascular complications of cancer therapy: incidence, pathogenesis, diagnosis, and management. J Am Coll Cardiol. 2009; 53:2231-2247.

27. Mikhail SE, Sun JF, Marshall JL. Safety of capecitabine: a review. Expert Opin Drug Saf. 2010; 9:831-841.

28. Dermitzakis EV, Kimiskidis VK, Eleftheraki A, Lazaridis G, Konstantis A. The impact of oxaliplatin-based chemotherapy for colorectal cancer on the autonomous nervous system. The impact of oxaliplatin-based chemotherapy for colorectal cancer on the autonomous nervous system. Eur J Neurology. 2004; 21:1471-1477.

\section{CORRESPONDÊNCIA}

Vitor Alexandre Pezolato

Rua Cincinato Braga, 306, apto 1101

Bela Vista, São Paulo - Brasil.

CEP: 01333-010

E-mail: vitor.pezolato@gmail.com 